\title{
Electron Beam Initiated Cross-linking in Hybrid Nylon 66 Membranes
}

\author{
A. W. Mohammad ${ }^{1 *}$, A. Linggawati ${ }^{2}$ \& Z. Ghazali ${ }^{3}$ \\ ${ }^{1 \& 2}$ Department of Chemical and Process Engineering, Universiti Kebangsaan Malaysia, 43600 UKM Bangi, \\ Selangor, Darul Ehsan, Malaysia \\ ${ }^{3}$ Malaysian Institute for Nuclear Technology Research (MINT), 43600 UKM Bangi, Selangor, Daeul Ehsan, \\ Malaysia
}

\begin{abstract}
Electron beam (EB) irradiation at dose of $80 \mathrm{kGy}$ in air has been used as a cross-linking initiator in hybrid nylon 66 membranes to improve the properties and performance of the membrane for separation processes. The cross-linking was characterized by gel content, swelling, scanning electron microscopy (SEM), and thermal stability. Membrane performance was investigated by pure water permeation and by rejection of raffinose and vitamin B12. The results showed that the EB irradiation successfully initiated cross-linking in hybrid nylon 66 membranes.
\end{abstract}

Keywords: Polymer, irradiation, effects, microstructure, thermooechanical effects

\subsection{INTRODUCTION}

Increased utilization of electron beam (EB) for the modification and enhancement of polymer properties has been well documented over the past forty years. EB processing has been demonstrated on a large commercial scale to be a very effective method for improving properties of various polymer materials. The EB irradiation can be used for scission of the polymer molecular chain, which subsequently leads to random rupturing of bonds and the cross-linking of polymer molecules. This process results in the formation of large, threedimensional molecular networks. In practice, chain scission and cross-linking can occur simultaneously, with either mechanism being predominant in some materials [1-3]. This

\footnotetext{
* Corresponding to: A. W. Mohammad (email: wahabm(a)eng.ulkm.my)
}

irradiation also results in significant modifications to the free volume and free volume distribution of the polymer matrix, which has significant impact on the permeability and permselectivity of the irradiated polymer. Various effects can be expected from this process.

Nylon 66, a polyamide (PA) derived from 1,6 hexamethylene diamine and adipic acid, contains a mixture of chains that have either amine or acid groups, or a combination of the two, at their ends. Reports in the literature have shown that the differences in end group configuration can lead to significant differences in the morphology [46]. Porous polyamide membrane has been commercialized for many years and is currently widely used in fine-separation processes $[7,8]$.

Study on radiation cross-linking of polyamide 66 (PA 66) or nylon 66 has been reported by several workers [9-10]. Nylon 66 is a typical EB 
irradiation cross-linkable semi crystalline polymer. Cross-linking can change the polymer properties in dramatic ways. For example, crosslinking has been shown to improve thermal properties, change polymer morphology and change the sieving characteristics of polymers.

Modified organic compounds (polymer) containing inorganic substrates have been termed "hybrid materials." In such hybrid materials, advantages found in one component can be used to complement the weaknesses in other components which constitute the hybrid material. Alkoxysilane is one inorganic material that is used to make hybrid materials. The concept of this modification is also to develop new and improved materials for application in the field of membrane science. Xu et al. [11] studied surface-modified nano- $-\mathrm{SiO}_{2}$ that was mixed with polyamide 66 by melt processing. Their result indicated that the incorporation of silica nanoparticles decreased crystallization temperature and increased the transition temperature of the composite. Kogure et al. [12] prepared an excellent organic-inorganic anion exchange capacity and electro-selectivity membrane by a liquid phase coupling method. Sol-gel is another popular and simple method that is used for the preparation of hybrid membranes. However, this method is not capable of producing good hybrid nylon 66 membranes. Our preliminary study found that the membrane is brittle. In view of this, EB irradiation was used to fabricate high performance membrane. It is known that $\mathrm{EB}$ irradiation can improve properties of materials.

In this study, hybrid membrane films were prepared from the polymerization of 3aminopropyltriethoxhysilane (APTES) in nylon 66 with EB as the initiator of reaction. Recently, Tezuka et al. (2007) prepared organic-inorganic membranes from 3-aminopropyltriethoxhysilane (APTES) and sulfuric acid. They found that the membrane is thermally stable up to $300^{\circ} \mathrm{C}$ in any composition.

The aim of this research was to investigate the effect of EB irradiation as a cross-linking initiator in hybrid nylon 66 membranes in order to improve the properties and performance of the membrane for separation. The membrane properties were characterized by gel content, degree swelling, scanning electron microscopy (SEM) and thermogravimetry analysis (TGA). The performance of the membrane for separation was investigated by pure water permeation and by the rejection of raffinose and vitamin $\mathrm{B} 12$.

\subsection{EXPERIMENT}

\section{$2.1 \quad$ Materials}

Additive-free Poly(hexametylene adipamide) (Nylon 66, product \# 42,917-1, pellet form) and 3-aminopropyltriethoxhysilane (APTES) (99\%) were purchased from Aldrich. Formic acid p.a (FA) (Merck, 99\%) was used as the solvent.

\subsection{Preparation of Membrane and EB Irradiation}

Nylon 66 pellets were initially dried in a vacuum oven at $60^{\circ} \mathrm{C}$ for $4 \mathrm{~h}$ and then thoroughly dissolved in the respective solvent at $12.5 \%$ concentration polymer $(\mathrm{w} / \mathrm{w})$ to form the first solution. APTES was mixed with distilled water and FA (1:4) for 1 $h$ as the second solution. The second solution was dispersed into the first solution under continuous agitation for $3 \mathrm{~h}$ (dope solution). The final composite solution of nylon 66/APTES had a 90/ 10 mass ratio and was labeled as $\mathrm{H} 10-0$. The homogeneous solution above was left for 24 hours to release air bubbles. A dope solution was uniformly spread on a glass surface using a casting Gardner knife as thin films (thickness ca. 350 $\mathrm{mm}$ ) and then irradiated by electron beam (EB, Alurtron NHV EPS-3000) at an acceleration voltage of $1 \mathrm{MeV}$ and a beam current $10 \mathrm{~mA}$. The irradiation dose was $80 \mathrm{kGy}$ and given in each pass, and the membrane was then labeled as $\mathrm{H} 10-$ 80 . The sample designations are given in Table 1.

\subsection{Characterization}

\subsubsection{Gel Content}

After irradiation, the films were vacuum dried at $80^{\circ} \mathrm{C}$ for $12 \mathrm{~h}(43,200 \mathrm{~s})$, and portions of the dried films were weighed. The dried film portions were then immersed in $85 \%$ formic acid. Continuous extraction was carried out for 3 days at room 
temperature (fresh solvent being added in little amounts every day); gels were collected by filtering through a fritted glass crucible. The percent gel content was calculated using the formula:

$$
\% \text { gel content }=\frac{\text { unextractable fraction }}{\text { irradiated film weight }} \times 100
$$

The results reported are the average of three measurements on three different portions of each film.

\subsubsection{Degree of Swelling}

The swelling of membranes in water was measured by immersing the dried membranes in water for $48 \mathrm{~h}$ at room temperature. The membrane was weighed only after the water on the surface of the swollen films was removed with cellulose paper. The dried membranes were obtained by drying at $60^{\circ} \mathrm{C}$ until they reached a constant weight. The degree of swelling $(\% \mathrm{~S})$ was defined by Equation (2), where $W_{s}$ is the weight of the swollen membranes and $W_{d}$ is the dried membrane weight.

$$
\% S=\frac{\left(W_{s}-W_{d}\right)}{W_{d}} \times 100
$$

\subsubsection{Morphological Properties}

Morphologies of membranes, including top and cross sectional views, were observed by using scanning electron microscopy, or SEM (Oxford Instrument, 7353, England). The cross section of the hybrid membranes was fractured in liquid nitrogen and coated with $\mathrm{Au}$ by sputtering.

\subsubsection{Thermal Gravimetric Analysis}

The thermogravimetric data were obtained from thermogravimetry TGA, pyris-1 (Perkin Elmer, USA) performed under dry nitrogen atmosphere at a flow rate of $30 \mathrm{ml} / \mathrm{min}$ and a temperature range of $40-600^{\circ} \mathrm{C}$ at a heating rate of $10^{\circ} \mathrm{C} / \mathrm{min}$. The samples were ground into a fine powder. The measurements were taken using 5-8 mg samples. Weight-loss against temperature curves was recorded.

\subsubsection{Permeation Measurement and Molecular Weight Cut-off}

Permeation measurement was carried out with distilled water, and molecular cut-off was determined by the rejection of raffinose and vitamin B12 (uncharged solutions). The solutions containing raffinose and vitamin B12 were prepared with distilled water at 500 ppm and 100 ppm, respectively. The concentrations of the vitamin $\mathrm{B} 12$ and raffinose solutions were analyzed using a spectrophotometer (Shimadzu UV$1601 \mathrm{PC})$. These solutions were thermostated at $25^{\circ} \mathrm{C} \pm 0.2{ }^{\circ} \mathrm{C}$ in a water bath for at least one hour before being used in the experiment. The membrane was immersed at least overnight in distilled water before being used in any experimental work. Solute rejection was determined based on $R_{\text {real }}$, defined by:

$$
R_{\text {real }}=1-\frac{C_{p}}{C_{w}}
$$

where, $\mathrm{C}_{p}$ is a concentration of permeate and $\mathrm{C}_{w}$ is a concentration of feed.

Permeation studies were carried out in an Osmonics SEPA@ST (dead-end) cell. It had a 300 $\mathrm{mI}$ volume capacity and effective membrane area of $15.2 \mathrm{~cm}^{2}$. The maximum operating pressure of the cell was 450 psig ( 31 bar). There was a magnetic stirrer assembly inside the cell feed chamber, which allowed for the use of magnetic stir plates for mixing. The stirrer's operating speed was $400 \mathrm{rpm}$, and the pressure of the experiment was 5-25 bar. Approximately $15 \mathrm{ml}$ of the permeate was collected in a graduated cylinder.

\subsection{RESULTS AND DISCUSSION}

\subsection{Gel Content and Degree of Swelling}

The gel content and degree of swelling analysis were carried out to investigate radiation crosslinking. Figure 1 shows the gel content and degree of swelling of the hybrid membrane nylon 66 before 


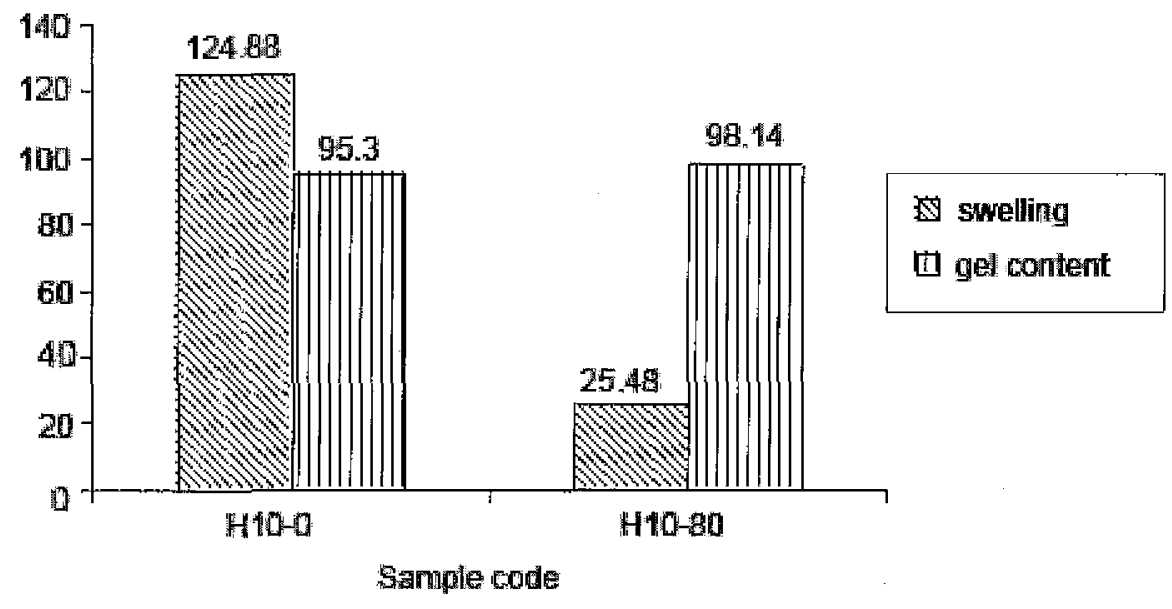

Figure 1 Gel content and degree of swelling of the hybrid membrane nylon 66 before and after EB irradiation at $80 \mathrm{kGy}$

and after EB irradiation at $80 \mathrm{kGy}$. From Figure 1, it can be seen that EB irradiation at $80 \mathrm{kGy}$ increased the gel content of hybrid nylon 66 and decreased the degree of swelling. These results indicate that the EB irradiation affected the radiation induced cross-linking network. At this dose, the trapped radicals accumulated causing an increase in the probability of recombination and, subsequently, more crosslinlings. The gel corresponds with the network structure in an amorphous region of nylon 66 due to the insolubility of the material. A previous study reported that EB-crosslinking increased gel content $[10,13,14]$. Sengupta et al. [10] also reported that, below $200 \mathrm{kGy}$, there is an absence of any gel content. They observed gel content starting at a dose of $200 \mathrm{kGy}$. Ueno [9] reported that PA 66 irradiated to a dosage of $60 \mathrm{kGy}$ by $\mathrm{EB}$ exhibited a gel content of $80 \%$, and, at double the radiation dose, the gel content was only slightly decreased. The relationship between gel content and radiation-induced crosslinking is well described by [2].

From Table 1, it can be seen that the increase of the gel content is not only induced by $\mathrm{EB}$ irradiation but also contributed to by APTES. In our previous work [15] before the addition of APTES, the gel content of nylon 66 only $30.93 \%$. As is well known, APTES is one of the known silane coupling agents.

\subsection{Scanning Electron Microscopy (SEM)}

Figure 2 illustrates the top surface and the cross section of hybrid nylon 66 membrane prepared by sol-gel and EB irradiation. It can be seen that the top surface of the membrane after irradiation exhibited a smooth surface (Figure 2(c)) as compared to before irradiation (Figure 2(a)). The cross section image of unirradiated hybrid membrane is very porous, making this membrane

Table 1 Designation and irradiation dose of dope solution

\begin{tabular}{lll}
\hline $\begin{array}{l}\text { Sample } \\
\text { code }\end{array}$ & $\begin{array}{l}\text { Nylon 66/ } \\
\text { APTES (part) }\end{array}$ & $\begin{array}{l}\text { Radiation dose } \\
\text { (kGy) }\end{array}$ \\
\hline H10-0 & $90 / 10$ & 0 \\
H10-80 & $90 / 10$ & 80 \\
\hline
\end{tabular}


unable to retain pure water and uncharged solute. It is clear from Figure 2 that the top surface of the nylon 66 membranes developed into a dense nonporous skin with EB irradiation. Formation of this dense non-porous skin is due to the sharp increase in interfacial polymer concentration from the EB treatment. Therefore, the top surface of H10-80 membrane is more dense (Figure 2c) and exhibits-smaller pore sizes (Figure 2d) when compared to H10-0, making it capable of rejecting raffinose and vitamin $\mathrm{B} 12$. The dense membrane was attributed to crosslinking reactions that form a three-dimensional network during the radiation process, and the membrane pore size thus becomes narrower than before irradiation [16]. Krajewska and Olech [16] also reported that crosslinking causes a reduction in pore size. In this study, the crosslinking reaction was supported by gel content and degree of swelling in water data as shown in Figure 1. Crosslinking will cause an increase in gel content and a reduction in the degree of swelling in pure water.

\subsection{Thermogravimetruc Analysis}

Thermogravimetric analysis was used to analyze the thermal stability of the material. Thermogravimetric analysis was undertaken on the hybrid nylon 66 membrane in order to determine the effect of $\mathrm{EB}$ irradiation on the thermal properties of the hybrid membrane. The thermograms of TGA -DTA are shown in Figs. 3 and 4 . These figures show that the temperature of $10 \%$ weight loss for the unrradiated hybrid membrane is lower than that for the radiated hybrid membrane, at 352.99 and $422.18^{\circ} \mathrm{C}$, respectively. This indicates that $\mathrm{EB}$ irradiation increased the thermal stability of the hybrid nylon 66 membrane. The unirradiated hybrid nylon 66 membrane has three degradation stages. The temperature of each degradation stage in the hybrids was attributed to scissions of the organic compounds, the end chain ( $n$-propyl segment) of the organosiloxanes and the imide segments [17]. The first degradation step of the hybrid membrane is at $173.5^{\circ} \mathrm{C}$ due to the incomplete hydrolysis and condensation reactions and different water and solvent adsorption ability. This was caused by a different organic-inorganic

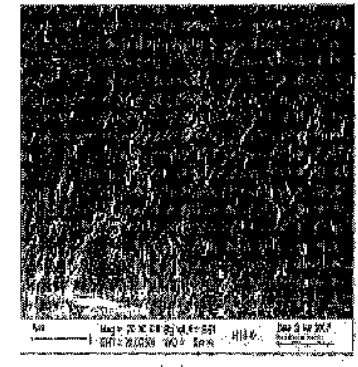

(a)

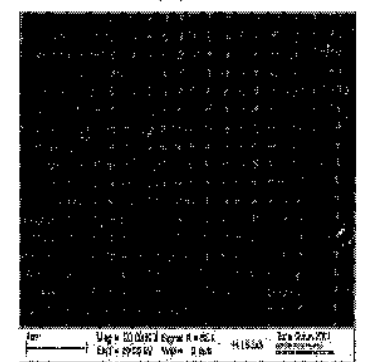

(c)

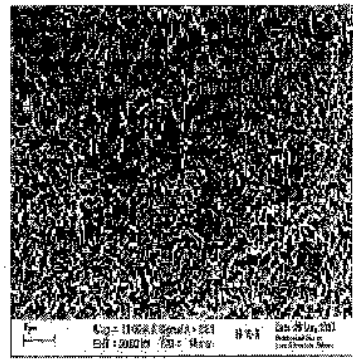

(b)

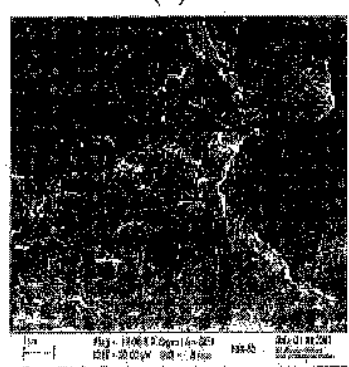

(d)

Figure 2 SEM of unirradiated hybrid membrane (H10-0), (a) top surface, (b) crosssection and irradiated (H10-80) (c) top surface dan (d) cross section

crosslink manner, as previously suggested by [18]. The weight loss of about $27.2 \%$ at the onset of degradation of the hybrid membrane is at $384.91^{\circ} \mathrm{C}$, with the maximum temperature at $423.04^{\circ} \mathrm{C}$, which occurs at the second degradation stage. According to Tezuka et al. [19], the weight loss observed at $400^{\circ} \mathrm{C}$ corresponds to the combustion of organic moieties. The decomposition in the temperature range 400$500^{\circ} \mathrm{C}$ was also ascribed to the decomposition of adhered PA 66 on the silica surface [11]. The last stage occurs at $477.44{ }^{\circ} \mathrm{C}$, with a weight loss of $50.7 \%$, corresponding to the decomposition of the main chains of nylon 66 .

Irradiated hybrid nylon 66 has only one degradation stage. The onset of degradation is $436.35^{\circ} \mathrm{C}$ at the peak maximum temperature of $480.2{ }^{\circ} \mathrm{C}$. This result shows that EB irradition increased the thermal stability and compatibility of the silane compound in the matrix polymer. Two reasons can be suggested as to why the thermal stability increased in this case. The first reason is due to Si-O-Si linkages in the hybrid membranes, 
which increased the degradation temperature by shifting the weight loss to a higher temperature $[15,19]$. The second reason is due to the crosslinking of alkyl, amide or amine groups in the irradiated hybrid membrane. These crosslinkages can be based on the crossliking of alkyl, amine or amide groups in the hybrid membrane or the Si-O-Si covalent bond. This will lead to the initial temperature of weight loss of the irradiated membrane being higher than that of unirradiated membrane, but, above $500^{\circ} \mathrm{C}$, the percent of weight loss of H10-80 is higher than $\mathrm{H} 10-0$. It is interesting to note that, above $500^{\circ} \mathrm{C}$, the weight loss of unirradiated hybrid membrane is lower than that of irradiated membrane. There are some reasons behind this occurance. One important reason is the presence of the additive in nylon 66 [20]. Radiation effects on the properties of a polymer are difficult to predict, especially in presence of certain additives [21]. Another reason might be due to the crosslink interaction between nylon 66 and APTES, even without the application of radiation. It is known that APTES is one of the silane coupling agents. Silane coupling agents are predominantly used as mediators that bind organic and inorganic materials and also reinforce plastics [22]. These results suggest that the thermal stability of hybrids was enhanced not only by EB irradiation but also by additive behaviour and silica content.

\subsection{Permeation Measurement}

Table 2 shows the permeation study of distilled water. As shown in the second column of the table, the water flux for non irradiated membranes
(H10-0) was not detected, indicating that this membrane did not exhibit water retention capability due to large menbrane pore diameters. Crosslinking made the membrane pore sizes narrower than before irradiation. As a result, water fluxes can be detected.

Water permeability of the membrane was determined by the gradient of water flux against pressure. The water permeability of the $\mathrm{H} 10-80$ membrane is $16.84 \mathrm{Lm}^{-2} \mathrm{~h}^{-1} \mathrm{bar}^{-1}$. Based on water permeability, irradiated hybrid nylon 66 at a dose of $80 \mathrm{kGy}(\mathrm{H} 10-80)$ is of nanofiltration type. The range of water permeability values for nanofiltration membranes that are commercially available is between $1.33 \mathrm{Lm}^{-2} \mathrm{~h}^{-1} \mathrm{bar}^{-1}$ and 50.50 $\mathrm{Lm}^{-2} \mathrm{~h}^{-1} \operatorname{bar}^{-1}[23]$.

\subsection{Determination of Molecular Cut-off (MWCO)}

Table 2 also shows the membrane solute rejection of vitamin B12 and raffinose at a pressure of 10 bar. As can be seen from this table, the rejection of vitamin B12 $(91.87 \%)$ is higher than raffinose (73.34\%) for the irradiated membrane. However, there is no rejection by non-irradiated membrane. Solute molecular size is taken into account as the factor in determining the rejection [24]. Vitamin B12 is a larger solute molecule when compared to raffinose, such that vitamin $\mathrm{B} 12$ has a higher rejection rate. Rejection occurs in pores smaller than the solute. These smaller pore sizes are due to cross-linking induced by EB irradiation. Membranes having smaller pore sizes exhibit phenomena such as convection and diffusion, which contribute to the separation process.

Table 2 Membrane permeation at $10 \mathrm{bar}$

\begin{tabular}{|c|c|c|c|c|}
\hline \multirow[b]{2}{*}{ Membrane code } & \multirow[b]{2}{*}{$\begin{array}{l}\text { Water flux } 10^{-6} \\
\left(\mathrm{~ms}^{-1}\right)\end{array}$} & \multirow[b]{2}{*}{ MWCO (Da) } & \multicolumn{2}{|c|}{ Rejection (\%) } \\
\hline & & & Raffinose & Vitamin B 12 \\
\hline $\mathrm{H} 10-0$ & nd & nd & nd & nd \\
\hline $\mathrm{H} 10-80$ & 46.79 & 1355 & 73.34 & 91.87 \\
\hline
\end{tabular}

nd $=$ no detected because no water flux was obtained 
Generally, these results clearly show that EB irradiation treatment affected the membrane molecular cut-off. The MWCO of the irradiated hybrid membrane is $1355 \mathrm{Da}$. Based on MWCO and rejection data of uncharged solute (vitamin $\mathrm{B} 12$ and raffinose), the irradiated hybrid nylon 66 at a dose of $80 \mathrm{kGy}(\mathrm{H} 10-80)$ is of nanofiltration type.

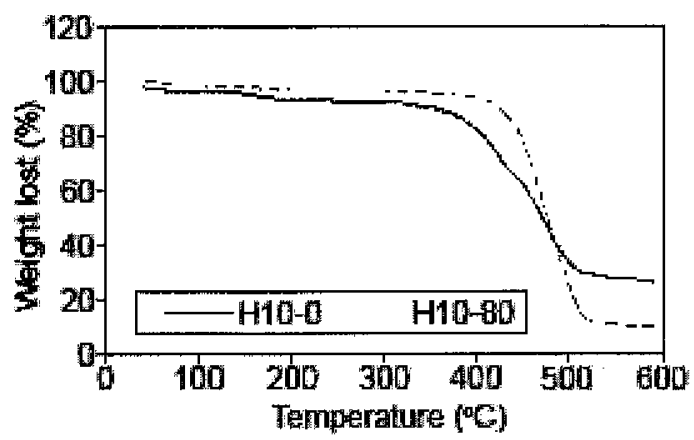

Figure 3 TGA curve of unirradiated (H10-0) and irradiated (H10-80) hybrid membranes

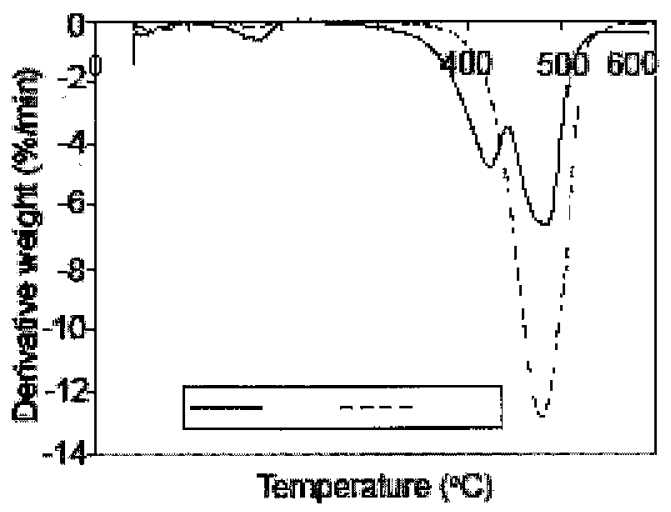

Figure 4 DTA curve of unirradiated (H10-0) and irradiated (H10-80) hybrid membranes

\subsection{CONCLUSION}

Based on the results from this study, it can be concluded that EB irradiation initiated crosslinking of hybrid nylon 66 membranes. The evidence of crosslinking was demonstrated by an increase of the gel content and decrease of the degree of swelling. The crosslinlking improved the performance of the hybrid membrane. From TGA analysis, it was found that irradiated hybrid membrane has a higher thermal stability than that of unirradiated membrane. Upon irradiation, membrane pore sizes become smaller and rejection of vitamin $B 12$ is high. Based on water permeability and rejection data of vitamin B12 and raffinose, irradiated hybrid nylon 66 membrane at a dose of $80 \mathrm{KGy}(\mathrm{H} 10-80)$ is of nanofiltration type.

\section{ACKNOWLEDGEMENT}

The authors would like to thank the Riau Province Government for scholarship assistance. We would also like to acknowledge EB Alurtron team Malaysian Institute for Nuclear Technology Research (MINT) for the collaboration.

\section{REFERENCES}

[1] Chmielewski, A. G., Haji-Saeid, M., 2004. Radiation Technology: Past, Present and Future. Radiation physics and Chemistry. 71: $17-21$.

[2] Charlesby, A. A., 1960. In Atomic Radiation and Polymers. London: Pergamon Press. 11: 72 .

[3] Silverman, J., 1977. Basic Concepts of Radiation Processing, Radiat. Phys. Chem. 9(1-3): 1- 15 .

[4] Oshinski, A. J., Kesklkula, H., Paul, D. R., 1992a. Rubber Toughening of Polyamides with Functionalized Block Copolymers 1. Nylon-6. Polymer. 33(2): 268-283.

[5] Oshinski, A.J., Keskkula, H., Paul, D. R., 1992b. Rubber Toughening of Polyamides with Functionalized Block Copolymers: 2 Nylon-6,6. Polymer. 33(2): 284-293.

[6] Takeda, Y., Keskkula, H., Paul, D. R, 1992. Effect of Polyamide Functionality on the Morphology and Toughness of Blends with a Functionalized Block Copolymer. Polymer. 
33(15): $3173-3181$.

[7] Persson, A., Jonsson, A. S., Zacchi, G., 2003. Transmission of BSA during Cross-flow Microfiltration: Influence of $\mathrm{pH}$ and Salt Concentration. J. Memb. Sci.223(1-2): 1121.

[8] Castilho, L. R., Anspach, F. B., Deckwer, W.D., 2002. Comparison of Affinity Membranes for Purification of Immunoglobulins. J. Memb Sci. 207(2): 253-264.

[9] Ueno, K., 1990. The Radiation Cross linking Process and New Products. Radiation Physics and Chemistry. 35(1-3): 126-131.

[10] Sengupta, R., Tiklku, V. K., Somani, A. K., Chaki, Tapan, T. K, Bhowmick, A. K., 2005. Electron Beam Irradiated Polyamide-66 Film-II: Mechanical and Dynamical Properties and Water Absorption Behaviour. Radiation Physics and Chemistry. 72(6): 751-757.

[11] Xu, X., Li,B., Lu, H., Zhang, J., Wang, H., 2007. The Interface Structure of Nano$\mathrm{SiO}_{2} /$ PA 66 Composites and Its Influence on Material's Mechanical and Thermal Properties. Applied Surface Science, 254: 1456-1482.

[12] Kogure, M., Ohya, H., Paterson, R., Hosaka, M., Kim, J-J., McFadzean, S., 1997. Properties of New Inorganic Membranes Prepared by Metal Alkoxide Methods Part II: New Inorganic-organic Anion-exchange Membranes Prepared by the Modified Metal Alkoxide Methods with Silane Coupling Agents. J. Memb. Sci. 126(1): 161-169.

[13] Djafar, G., Behjat, A., 2001. Radiation Crosslinking of LDPE and HDPE with 5 and $10 \mathrm{MeV}$ Electron Beams. European Polymer Journal. 37: 2011-2016.

[14] Södergård, A., Ekman. K., Stenlund, B., Lassas, A-C., 1996. The Influence of EBCrosslinking on Barrier Properties of HDPE-mica Composite.J. App. Polym. Sci. 59(11): 1709-1714.

[15] Linggawati A, .Mohammad AW, Gazali Z. Modified Surface Properties of Hybrid Nylon 66 Membrane by EB Irradiation,
Proceeding of $3^{\text {rd }}$ Scientific Conference, Bangi, Malaysia, January, 2008.

[16] Krajewski,.B., Oleich,A., 1996. Pore Structure of Gel Chitosan Membranes. I. Solute Diffusion Measurements. Polymer Gel Network. 4(1): 33-43.

[17] Chakrabarti, K., Kim, S. M., Oh, E. O., Whang, C. M., 2002. Thermal Analysis of Poly(dimethyl-siloxane)-modified Silica Xerogels. Materials Letters. 57(1): 192-197.

[18] Zong, S -H., Li, C-F., Xiao X-F., 2002. Preparation and Characterization of Polyimide-silica Hybrid Membranes on Kieselguhr-mullite Supports. J. Memb.Sci. 199(1-2): 53-58.

[19] Tezuka, T., Tadanaga, K., Ayashi, A., Tatsumisago, M., 2007. Inorganic-organic Hybrid Membranes Prepared from 3aminoprophyltriethoxysilane and Sulfuric Acid as Anhydrous Proton Conductor. Solid State Ionics. 178: 705-708.

[20] Linggawati, A., Mohammad, A. W, Gazali, Z., Preparation of Nylon 66 Membrane by Electron Beam Irradiation: Dynamic Mechanical Properties and Water Absorption Behaviour. Proceeding of Regional Symposium on Chemical engineering. Yogyakarta, Indonesia, December 2007.

[21] Cleland, M. R., Parks, L. A., Cheng, S., 2003. Applications for Radiation Processing of Materials. Nuclear Instrument and Methods Physics Research. B 208: 66-73.

[22] Jio, C., Wang, Z., Gui, Z., Hu, Y., 2005. Silane Grafting and Crosslinking of Ethyleneoctene Copolymer. European Polymer Journal. 41: 1204-1211

[23] Bowen, W. R., Mohammad, A. W., 1998. Characterization and Prediction of Nanofiltration Membrane Performance a General Assessment. Trans.IChemE. 76(A): 885-893

[24] Tsui, E. M., Cheryan, M., 2004. Characteristics of Nanofiltration Membranes in Aqueous Ethanol. J. Memb.Sci. 237(1-2): 61-69. 